\title{
A Staggered-Mesh Finite-Difference Numerical Method for Solving the Transport Equations in Low Pressure RF Glow Discharges
}

\author{
Michael S. Barnes, Tina J. Cotler, and Michael E. Elta \\ Solid State Electronics Lab, University of Michigan, Ann Arbor, Michigan 48019
}

Received May 5, 1987; revised September 9, 1987

\begin{abstract}
A numerical model of a low pressure parallel plate if glow discharge is presented based on a self-consistent formulation of the energy-momentum conservation equations for electrons, the continuity equations for both electrons and ions, and Poisson's equation. Various explicit finite-difference numerical methods are discussed in terms of stability and overshoot properties. Stability considerations for the numerical method that was implemented, including the initial and the boundary conditions, are examined. Results from a large-signal simulation of a low pressure argon of glow discharge are presented. (c) 1988 Academic Press, Inc.
\end{abstract}

\section{INTRODUCTION}

Over the last decade, plasma processing of semiconductor materials has achieved widespread popularity in the microelectronic industry. Low pressure parallel plate If glow discharges at $13.56 \mathrm{MHz}$ are used to generate chemically reactive gas phase species in plasma etching [1] and plasma-enhanced chemical vapor deposition [2]. Moreover, these discharges can provide a means of physically bombarding a semiconductor surface with "heavy" positive ions, thereby enabling sputtering and anisotropic plasma etching [3]. As the industry focuses on process automation, the need to quantitatively understand some of the fundamental components of integrated circuit manufacturing becomes increasingly important. Since many of the physical and chemical processes are analytically too complex, it appears necessary to develop accurate numerical models for such purposes.

An $\mathrm{rf}$ glow discharge is a weakly ionized plasma which under ordinary conditions does not reach a state of local thermodynamic equilibrium. The "hot" electrons are responsible for ionization, excitation, dissociation, etc., of the "cold" neutral gas atoms and/or molecules, resulting in a self-sustaining discharge that produces chemically reactive gas phase species at temperatures far lower than otherwise possible. More specifically, the electrons are heated by the if electric field and can lose significant amounts of energy only through inelastic collisions. Consequently, the electrons must heat to energies above inelastic thresholds before cooling can 
occur. On the other hand, the ions remain cool since they continually transfer energy and momentum to neutrals through elastic collisions.

The electrical properties of an $\mathrm{rf}$ glow discharges are primarily attributed to highly nonlinear carrier dynamics, electrode sheath effects, and interaction with an external circuit (i.e., a power source and matching network). Since the electrons cannot transfer significant energy and momentum to neutrals during elastic collisions (due to the high neutral to electron mass ratio), it becomes important to consider electron momentum and energy effects when modeling rf glow discharges. Self-consistent numerical models $[4,5]$ based on the electron and ion continuity equations and Poisson's equation do not account for these effects. The continuum model [6], which includes an energy balance equation, loses its potential for properly modeling the electron energy effects by assuming constant electron mobility, diffusivity, and ionization coefficient. The only nonlinear coupling between the electron energy balance equation and continuity equations in this model occurs through the generation rate term which is simply the ionization coefficient times an exponential containing the electron energy.

A fully comprehensive numerical model of an $\mathrm{rf}$ glow discharge must take into account transport properties under dynamic conditions. This could in theory be accomplished using Monte Carlo methods or by solving the Boltzmann equation. Monte Carlo methods have been used to model electron swarms [7-9] and more recently electron properties in if glow discharges [10]. Numerical solutions of the Boltzmann equation based on spherical harmonic expansions have also been developed [11,12]. While these approaches are quite useful for specific applications, none are capable of self-consistently modeling rf glow discharges due to the extreme computational complexity that is involved. In this paper, a transport model of an rf glow discharge is presented which can be self-consistently solved (numerically) using explicit finite-difference methods for compressible inviscid fluids in one dimension on a staggered mesh. The numerical method used is outlined and compared with other explicit finite-difference schemes in terms of stability and accuracy. Finally, some large-signal simulation results are presented for a $300 \mathrm{mT}$ argon $\mathrm{rf}$ glow discharge at $13.56 \mathrm{MHz}$, illustrating some of the physics of these discharges.

\section{The Transport Model}

The starting point for the rf glow discharge model is to assume the Boltzmann equation accurately describes particle transport [13]. This equation is given by

$$
\frac{\partial f}{\partial t}=-\bar{v} \cdot \nabla_{\bar{r}} f-\frac{\bar{F}}{m} \cdot \nabla_{\bar{v}} f+\left(\frac{\partial f}{\partial t}\right)_{\text {collisions }},
$$

where $f$ is the distribution function, $t$ the time, $\bar{r}$, the position $\bar{v}$ the velocity, $\bar{F}$ the external force, and $m$ the particle mass. The transport model is derived by taking 
moments of Eq. (1) and making certain simplifying assumptions [14]. The carrier density, the mean carrier momentum, and the mean carrier energy are defined by the zeroth, first, and second velocity moments of $f$,

$$
\begin{aligned}
n(\bar{r}, t) & =\int f(\bar{r}, \bar{v}, t) d^{3} v, \\
m n(\bar{r}, t) \bar{u}(\bar{r}, t) & =\int m \bar{v} f(\bar{r}, \bar{v}, t) d^{3} v, \\
n(\bar{r}, t) w(\bar{r}, t) & =\int \frac{1}{2} m \bar{v}^{2} f(\bar{r}, \bar{v}, t) d^{3} v,
\end{aligned}
$$

where $n$ is the carrier concentration, $\bar{u}$ the average velocity, and $w$ the average energy.

The one-dimensional forms of the first three moment equations for electrons are given by

$$
\begin{aligned}
& \frac{\partial n}{\partial t}=-\frac{\partial(u n)}{\partial x}+\alpha n, \\
& \frac{\partial u}{\partial t}=-u \frac{\partial u}{\partial x}-\frac{q E}{m}-\frac{1}{n m} \frac{\partial P}{\partial x}-\frac{u}{\tau_{v}}, \\
& \frac{\partial w}{\partial t}=-u \frac{\partial w}{\partial x}-q u E-\frac{1}{n} \frac{\partial(u P)}{\partial x}-\frac{w-w_{0}}{\tau_{w}} .
\end{aligned}
$$

Equation (5) is the usual form of the particle continuity equation where $n$ represents the electron density, $u$ the electron average velocity in the $x$ direction, $t$ the time, and $\alpha n$ the approximate form for the zeroth moment of the collision integral. Similarly, Eqs. (6) and (7) denote the standard forms of the per carrier average velocity (i.e., momentum) and energy transport equations. In these equations, $w$ is the average electron energy, $E$ the electric field in the $x$ direction, $m$ the electron mass, $P$ the electron pressure, and $w_{0}$ the thermal energy associated with the neutral gas reservoir (i.e., $0.0258 \mathrm{eV}$ ). The first and second moments of the collision terms are approximated by relaxation terms containing the effective momentum and energy relaxation time, i.e., $\tau_{v}$ and $\tau_{w}$, respectively. The equation of state for an ideal gas $P=n k_{b} T$ is used to define the electron pressure $P$ where $k_{b}$ is Boltzmann's constant and $T$ the electron temperature. In keeping with the usual concept of temperature, the thermal component of $w$ is defined by

$$
\frac{3}{2} k_{b} T=w-\frac{1}{2} m u^{2}
$$

and the pressure term reduces to

$$
P=\frac{2}{3} n\left(w-\frac{1}{2} m u^{2}\right) .
$$


A more complete derivation of the first three moments of Boltzmann equation (commonly known as the conservation equations) can be found in a number a references, e.g., see Refs. [14-17].

The ion transport can be accounted for using a simpler model (for computational efficiency) when it is assumed that the ions are in equilibrium with the electric field. This transport model includes the continuity equation,

$$
\frac{\partial p}{\partial t}=-\frac{1}{q} \frac{\partial J_{p}}{\partial x}-\alpha n,
$$

where $p$ is the ion density and $q$ the electronic charge. The ion flux (i.e., the density times the average velocity) has been replaced by an expression for the ion current given by

$$
J_{p}=q v p-q D \frac{\partial p}{\partial x} .
$$

which includes a field-driven drift term and a gradient-driven diffusion term. In Eq. (11), $v$ is the ion drift velocity, and $D$ the ion diffusion coefficient. This transport model has been used quite extensively in glow discharge theory, e.g., see Ref. [18-20], and can be derived in a very straightforward manner from the conservation equations when certain simplifying assumptions are valid.

The effects of space charge are accounted for by including Poisson's equation,

$$
\frac{\partial E}{\partial x}=\frac{q}{\varepsilon_{0}}(p-n),
$$

in the transport model where $\varepsilon_{0}$ is the permittivity of free space. To obtain a large-signal solution, the system of nonlinear coupled partial differential equations, including Eqs. (5)-(7), (10) and Poisson's equation, must be solved self-consistently. In Section IV, a numerical method is presented for this purpose.

\section{The Collision Term and Transport Coefficients}

For a collision-dominated transport model formulated by taking successive moments of the Boltzmann equation, the accuracy by which the model describes the true physical situation is largely dependent on the forms used to approximate the moments of the collision integral. The approach utilized in this study is to use first-order terms which suitably approximate the rates by which $n, p, u$, and $w$ are changing due to collision processes. In accordance with Ref. [21], a three-level model consisting of a ground state, an excited state with $\varepsilon_{1}=11.60 \mathrm{eV}$ (representing the first four excited states $P_{2}^{3}, P_{1}^{3}, P_{0}^{3}$, and $P_{1}^{1}$ with threshold energies $11.55,11.62$, 11.74 , and $11.83 \mathrm{eV}$, respectively), and an ion ground state with $\varepsilon_{2}=15.76 \mathrm{eV}$. The total cross section data as a function energy has been obtained from standard sour- 
ces $[22,23]$. Electron-electron, electron-ion, and electron-metastable collisions are neglected since at low pressures the degree of ionization is typically $10^{-6}$ to $10^{-4}$ and the degree of excitation about $10^{-3}$ to $10^{-2}$ in an argon of glow discharge at $13.56 \mathrm{MHz}$ [3]. It is also reasonable to assume that attachment processes and three-body recombination (except at the electrode surfaces) can be neglected in an argon discharge. The problem of approximating the collision integral is thereby reduced to calculating $\alpha, \tau_{v}$, and $\tau_{w}$ as a function of the average electron energy and $v$ and $D$ as a function of the electric field.

To formulate data sets for electron collision parameters, the Boltzmann equation is solved under "uniform" dc conditions where the dc electric field is varied each time a solution is reached until a large enough number of solutions are available to approximate functional curves (with average energy as the independent axis.). The method of solution used for this purpose is a single particle Monte Carlo simulation described in detail in Ref. [5]. Under "uniform" conditions (i.e., all spatial and temporal gradients are zero), Eqs. (6) and (7) reduce to

$$
\tau_{v}=\frac{m u}{q E}
$$

and

$$
\tau_{n}=\frac{w-w_{0}}{q u E}
$$

closed form terms for the relaxation times [14]. These effective relaxation times are analogous to those used by Blotekjaer [24] for electron transport in semiconductors. The primary justification for using these forms is based on the assumption that
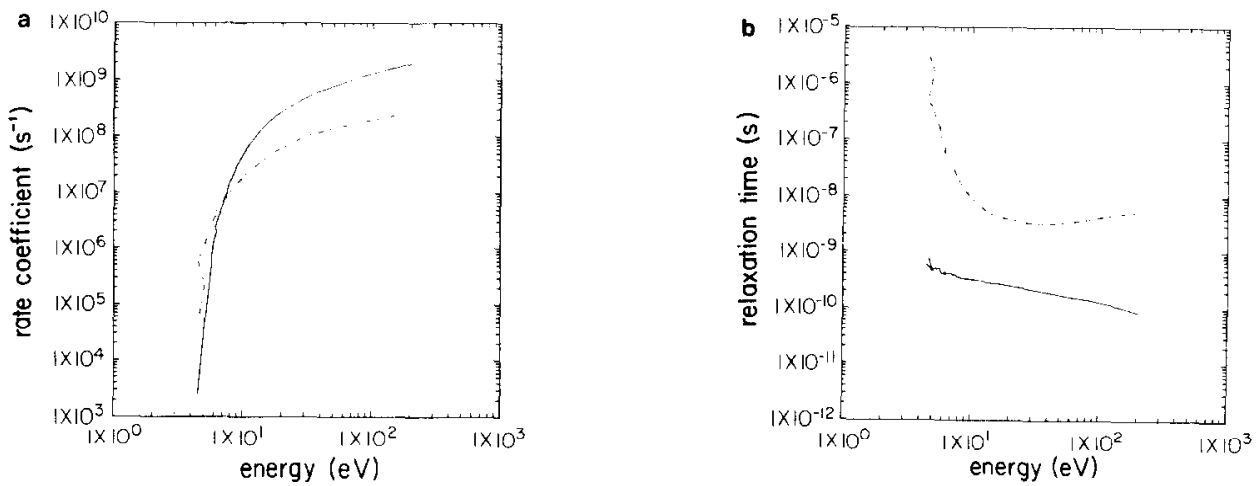

FIG. 1. Monte Carlo transport and rate data for electrons in argon at $300 \mathrm{mT}$ : (a) Electron impact rate coefficients vs average electron energy. The solid line indicates ionization and the dashed line excitation. (b) Effective relaxation times vs average electron energy. The solid line indicates momentum and the dashed line energy. 
the distribution function is approximately isotropic in velocity space (i.e., the thermal energy is much greater than the drift energy).

The average velocity and average energy are direct results of the Monte Carlo simulation and $\alpha$ is calculated by integrating the total ionization cross section times the velocity with the distribution function. For the data plotted in Figs. 1a and b, the dc electric field was varied from 1 to $500 \mathrm{~V} / \mathrm{cm}$ for an electron in argon at $300 \mathrm{mT}$. In the actual large signal computer program described later in this paper, these values are smoothed and stored in look-up tables for computational efficiency. Intermediate values between data points are determined by interpolation. Data curves for argon ion drift velocity and diffusivity at $300 \mathrm{mT}$ as a function of the electric field using this method are presented in Ref. [5].

\section{The Numerical Model}

\section{IV.A The Basic Scheme}

The transport model, i.e., Eqs. (5)-(7), (10), and (12), cannot be solved analytically without drawing on assumptions that are not valid under normal rf glow discharge operating conditions. With this in mind, a suitable alternative is to incorporate finite-difference numerical methods into a computer simulation. Hence, a numerical method must be implemented which is computationally accurate, stable, and efficient. Due to stability problems which occur as a result of source terms, relaxation terms, boundary conditions, and initial conditions, most commonly used finite-difference numerical methods in fluid dynamics [25] which appear to be suitable for this purpose cannot be used.

The primary stability problem arises in the numerical solution of the three equations governing electron transport. These coupled equations tend to oscillate close to the boundary points regardless of the numerical method used. Several numerical methods from fluid dynamics (for solving inviscid compressible flow equations) were unsuccessfully implemented. These include standard upwind differencing [25], Lax's method [26], a two step Lax-Wendroff method [27] and MacCormack's method [28]. The Lax-Wendroff and MacCormack methods reached a steady-flow solution when artificial explicit duffusion of the Longley type [29] was included. Since neither of these methods possess the transportive property [25], excessive wiggles in the solution propagated throughout the region of simulation, removing the effects of the sheath regions (i.e., to behave as capacitances which give rise to displacement current in the terminal discharge characteristics). More of the details pertaining to this type of nonlinear instability will be discussed in Sectin IV.C.

The numerical method used for the electron transport equations is a modified form of upwind differencing utilizing a staggered space grid. This particular mesh system is depicted in Fig. 2, where certain dependent variables are defined on each set of space points. Throughout this section, the ion/electron densities will be 


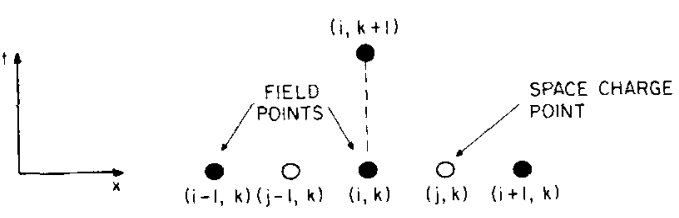

FIG. 2. Finite-different space-time diagram.

defined on the points with space index $j$ whereas all other dependent variables (i.e., $u, w$, and $E$ ) and parameters which are functions of these quantities will be defined on points with the space index $i$. The $i$ and $j$ space indices will be referred to by variable subscripts and the timestep index $k$ by a variable superscript in the finitedifference notation.

Throughout the evolution of the finite-difference scheme used for the electron transport equations, the damping of certain nonlinear instabilities seemed to be the most elusive problem to solve. To obtain a usable solution, all advected terms require finite difference operators that are transportive and all terms that are in conservation form must be conservatively difference. A numerical scheme has been implemented which utilizes the staggered mesh system and the following finitedifference operators:

1. An upwind difference operator originally developed for two-terminal semiconductor device simulations [30] which is applied to the flux gradient term in the continuity equation. This operator is an offshoot of the second-order accurate "donor cell" method [31] and possesses both the conservative and the transportive properties.

2. The "donor cell" method [31] or second form of upwind differencing [25] for the velocity gradient term in the velocity equation and the resistive loss (or flow-work) term in the energy equation. Although this operator is conservative, it can be applied to the velocity gradient term (which is in nonconservative form) by changing $u(\partial u / \partial x)$ to its equivalent form $\frac{1}{2}\left(\partial\left(u^{2}\right) / \partial x\right)$. This method is also transportive.

3. A space-centered difference operator is applied to the pressure gradient term in the velocity equation. Since this operator is conservative but not transportive, pressure gradient effects are felt upstream as would be physically expected [25].

4. A standard upwind difference operator, [32] which is transportive but not conservative during velocity reversals, for the energy gradient term in the energy equation.

Using the notation previously discussed, the finite-difference form of the electron transport model can be written as 


$$
\begin{aligned}
& \frac{n_{j}^{k+1}-n_{j}^{k}}{\Delta t}=\frac{1}{q} \frac{J N_{i+1}^{k}-J N_{i}^{k}}{\Delta x}+\alpha_{j}^{k} n_{j}^{k} \\
& \frac{u_{i}^{k+1}-u_{i}^{k}}{\Delta t}=-\frac{1}{2} \frac{u_{j}^{k} u_{R}^{k}-u_{j-1}^{k} i_{L}^{k}}{\Delta x}-\frac{q E_{i}^{k}}{m}-\frac{1}{n_{i}^{k} m} \frac{P_{i+1}^{k}-P_{i-1}^{k}}{\Delta x}-\frac{u_{i}^{k+1}}{\tau_{v_{i}}^{k}} \\
& \frac{w_{i}^{k+1}-w_{i}^{k}}{\Delta t}=-u_{i}^{k} \frac{w_{I}^{k}-w_{l-1}^{k}}{\Delta x}-q u_{i}^{k} E_{i}^{k}-\frac{1}{n_{i}^{k}} \frac{u_{i}^{k} P_{R}^{k}-u_{j-1}^{k} P_{L}^{k}}{\Delta x}-\frac{w_{i}^{k+1}-w_{0}}{\tau_{w_{i}}^{k}},
\end{aligned}
$$

where $\Delta t$ is the timestep and $\Delta x$ is the spacestep. In Eq. (15), JN is the total electron current density defined on the $i$ th point by

$$
\begin{aligned}
J N_{i}^{k} & =-q n_{j}^{k} u_{i}^{k}, & & u_{i}^{k}<0 \\
& =-q n_{j-1}^{k} u_{i}^{k}, & & u_{i}^{k} \geqslant 0 .
\end{aligned}
$$

The ionization rate $\alpha$ is approximated with a first-order term and computed as a function of the average energy (using a look-up table for efficiency) on the $i$ space points and then averaged between adjacent points to determine the value at the $j$ th point. In Eq. (16), the quantity $u_{j}^{k}$ is determined by averaging between the values at points $i$ and $i+1$; similarly, the quantity $n_{i}^{k}$ is determined by averaging between the values at points $j$ and $j-1$. The subscripts $L$ and $R$ are indexes (also used in Eq. (17)) determined by

$$
\begin{aligned}
R & =i & u_{j}^{k} & \geqslant 0 \\
& =i+1 & u_{j}^{k} & <0 \\
L & =i-1 & & u_{j-1}^{k} \geqslant 0 \\
& =i & & u_{j-1}^{k}<0 .
\end{aligned}
$$

The pressure at the $i$ th point is defined by

$$
P_{i}^{k}=\frac{2}{3} n_{i}^{k}\left[w_{i}^{k}-\frac{m}{2}\left(u_{i}^{k}\right)^{2}\right]
$$

The index $l$ in Eq. (17) is equal to $i$ when $u_{i}^{k} \geqslant 0$ and $i+1$ when $u_{i}^{k}<0$. Finally, the energy and momentum relaxation terms are computed using a first-order, advanced-time approximation as a function of the average energy (using table look-ups). It has been shown [14] that this method of approximation improves stability and eliminates a limitation on the timestep (i.e., that the timestep must be smaller than half of the smallest relaxation time).

The finite-difference form of the ion drift-diffusion equation has already been discussed in Ref. [5]. In brief, a two-step method using upwind differencing for the drift term and a fully implicit difference operator for the diffusion term has proven to be very successful. The first step, i.e., the explicit step, is identical to Eq. (15) with the exception that the current is the ion drift current rather than the total ion 
current. The second or implicit step applies a tridiagonal matrix solver for numerical efficiency to the diffusion term. In finite-difference form, the ion "driftdiffusion" continuity equation becomes

$$
q \frac{p_{j}^{k+1}-p_{j}^{k}}{\Delta t}=-\frac{J P_{i+1}^{k}-J P_{i}^{k}}{\Delta x}-\frac{J P D_{i+1}^{k+1}-J P D_{i}^{k+1}}{\Delta x}+\alpha_{j}^{k} n_{j}^{k},
$$

where the ion drift and diffusion currents are respectively given by

$$
\begin{aligned}
J P_{i}^{k} & =q p_{j}^{k} v_{i}^{k}, & & E_{i}^{k}<0 \\
& =q p_{j-1}^{k} v_{i}^{k}, & & E_{i}^{k} \geqslant 0,
\end{aligned}
$$

and

$$
J P D_{i}^{k+1}=-q D_{i}^{k} \frac{p_{j}^{k+1}-p_{j-1}^{k+1}}{2 \Delta x}
$$

The ion drift velocity $v$ and the ion diffusivity $D$ are computed as functions of the electric field using table look-ups.

The one-dimensional form of Poission's equation is computed in a very straightforward manner using a forward difference to approximate the space differential given by

$$
\frac{E_{i+1}^{k}-E_{i}^{k}}{\Delta x}=\frac{q}{\varepsilon_{0}}\left(p_{j}^{k}-n_{j}^{k}\right)
$$

This relationship determines the relative electric field values at each $i$ space point. The absolute values of the electric field must be determined by the boundary conditions which are discussed in the next section.

\section{IV.B. Initial and Boundary Conditions}

A system of partial nonlinear differential equations requires the accurate specification of the initial and the boundary conditions for a correct solution. In a finite-difference numerical model, this is further complicated by the introduction of certain destabilizing numerical artifacts even when the physical problem is suitably described. For the transport model outlined in Section IV.A, five independent sets of boundary conditions on $n, u, w, p$, and $E$ or some dependent variables must be specified for time advancement. The initial conditions at $t=0$ include the values of the five dependent variables as functions of distance.

The boundary conditions at the nonemitting electrodes must include the effects of outflow and electrode charging. Moreover, in order to satisfy Gauss's law at all times charge must be strictly conserved. For the electron transport quantities, $n, u$, and $w$, the boundary conditions are set on the electron current and the pressure. First, the slope of the electron pressure at the electrode is set to zero requiring the 
slopes of the electron transport quantities to be independently set to zero. This type of Neumann boundary condition [25] is suitable for $u$ and $w$ but it allows for electron inflow (which does not physically occur when the electrodes are nonemitting). Therefore, this condition is modified by setting the electron current to zero when the velocity is in the inflow direction. Similarly, the boundary condition for the ion density is set so that no ion drift current can flow into the discharge. The slope of the ion density at the electrodes is set to zero so that there is no ion diffusion current at the electrodes. Mixed boundary conditions of this type are often referred to as Robbin's boundary conditions [25].

The finite-difference form of Poisson's equation (i.e., Eq. (23)) only determines the relative values of the electric field. The absolute values are determined by the boundary conditions which include the effects of an equivalent external circuit and the charge on the electrodes. The electrodes are assumed to be coated with a $100 \mu \mathrm{m}$ layer of an oxide with a relative dielectric coefficient $K$ of 4.0. Subsequently, the relative field level $E_{d}$ in the insulating coating is calculated using the relationship between the surface charge and the normal electric fields [33] given by

$$
K E_{d}-E=\frac{\rho_{s}}{\varepsilon_{0}},
$$

where $\rho_{s}$ is the surface charge density stored at each timestep. Once all relative field values have been computed, the absolute levels are determined using an equivalent external circuit of an rf power source and matching network. The circuit depicted in Fig. 3 is suitable for this purpose where it is asumed that an optimum match has been achieved. In brief, the circuit-discharge interaction at each timestep is computed in the following manner:

1. The relative field is integrated across the discharge to determine the instantaneous if voltage.

2. The total discharge instantaneous of current $I_{\text {tot }}$ is calculated using a discrete form of

$$
I_{\mathrm{tot}}(t)=\frac{A}{W} \int_{0}^{W}\left(J N_{\mathrm{tot}}+J P_{\mathrm{tot}}+\varepsilon \frac{\partial E}{\partial t}\right) d x,
$$

where $A$ is the discharge area.

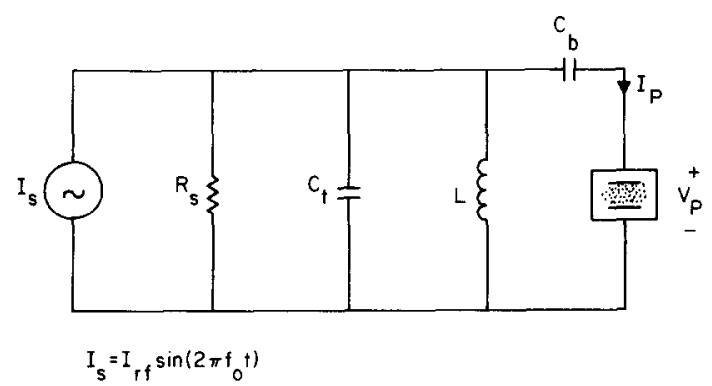

FIG. 3. Equivalent external circuit for boundary condition. 
3. The true discharge voltage is computed by using the value of $I_{\text {tot }}$ and the stored values of the inductance current and the capacitance voltages from the previous timestep.

4. The electric field is adjusted so that the true voltage equals the instantenous voltage.

The proper specification of the initial conditions is of fundamental importance to the convergence exhibited by the numerical method described in this paper. When the rf power is below a certain level, the constraints on the initial conditions are less restrictive. In this case, the electron energy and the particle densities are set to spatially uniform levels; the space charge and the electron velocity are set to zero across the discharge; the terminal voltage is set to zero and no charge is assumed to reside on the electrodes. On the other hand, this type of initial condition is unsuitable once the of power surpasses a certain level. One logical alternative then would be to use the values of all the dependent variables from a steady state solution at a lower of power as a starting point for a higher power solution. Fortunately, a steady state solution for a particular set of input conditions can be obtained at a reasonable power level using the less restrictive conditions. This solution is then used as the starting point for higher power solutions and the process can be repeated if necessary.

\section{IV.C. Stability Considerations}

The development of nonlinear instabilities in the finite-difference forms of the electron transport equations poses a serious problem when calculations involving a large number of iterations are necessary (e.g., a steady state solution). MacCormack has identified a particular nonlinear instability arising from the loss of sign information in the velocity flux gradient term of the velocity transport equation [28]. In an rf glow discharge at $13.56 \mathrm{MHz}$, the average electron velocity reverses direction twice during each $\mathrm{rf}$ cycle in a nonlinear manner (i.e., the velocity at each meshpoint does not change sign during the same timestep). Therefore, a situation can occur whereby a quantity of negative momentum can be transported out of a meshpoint containing only positive momentum, resulting in the formation of undamped oscillations in the spatial velocity profile. The use of the "donor cell" method for the finite differencing of this term alleviates this problem.

Another nonlinear instability in the electron transport finite-difference equations develops when the numerical boundary conditions are improperly specified. For this particular problem, the average energy is observed to become negative during velocity reversals at the second meshpoint from the electrode. Setting the electron pressure and current boundary conditions described in Section IV.B forces the finite-difference equations to permit average energy outflow only as electrons are absorbed by the electrodes. Other choices of numerical boundary conditions have been observed to violate the physical situation.

A linearized stability analysis based on the study of the amplification of Fourier components of the solution by the difference method $[14,25,28]$ is not included 
here since it does not predict any of the instabilities that develop. This type of analysis does however impose a limitation on the timestep equivalent to the Courant-Friedrichs-Lewy (CFL) condition for a compressible inviscid fluid [25]. In other words, there is a stability constraint on the speed at which a pressure disturbance can propagate (i.e., a pressure disturbance can propagate no more than one meshpoint per timestep). This is given by

$$
\Delta t \leqslant \Delta x /\left(|u|+\left[\frac{10}{9 m}\left(w-\frac{1}{2} m u^{2}\right)\right]^{1 / 2}\right),
$$

and reduces to the incompressible form when the average velocity is replaced by the drift velocity and the pressure term is omitted [5].

The timestep is also limited by a space charge stability condition and an electric field stability condition. A space charge instability usually occurs when the electric field is low, the particle densities are high, and the space charge is almost neutral and uniform [4]. When solving the continuity equations numerically, it is assumed that the rate of change of net charge is constant during a timestep and follows the tangent line of a decaying exponential curve. To prevent the net charge from changing sign during a timestep, the timestep must be less than the dielectric relaxation time, i.e.,

$$
\Delta t \leqslant \varepsilon_{0} / \sigma,
$$

where $\sigma$ is the conductivity. Similarly, a field instability usually occurs where there are large particle gradients, such as those encountered in the electrode sheaths. When this instability occurs, the net space charge accumulated during $\Delta t$ will be so large as to make $E$ overshoot its true value. This results in the development of undamped oscillations in subsequent timesteps. The timestep limitation which must be satisfied to eliminate this problem is also given by Eq. (27).

\section{IV.D. Large-Signal Simulation Results}

In order to obtain large-signal results, the external equivalent circuit values and the discharge parameters must first be specified. The circuit values are chosen so that the parallel tank circuit in Fig. 3 is resonant at $13.56 \mathrm{MHz}$. The blocking capacitance $C_{b}$ and the tank capacitance $C_{t}$ are set to $100 \mathrm{pF}$ and the tank inductance $L$ to $1.378 \mu \mathrm{H}$. Other combinations of $L, C_{t}$, and $C_{b}$ are feasible but numerical stability and computational stability are sacrificed to some degree. It has been observed that increasing the capacitances increases the computation time necessary to reach a steady state, whereas decreasing the capacitances can cause the simulation to go unstable. The source resistance $R_{s}$ is arbitrarily set to $1 \mathrm{M} \Omega$ to minimize its power dissipation. (The complete omission of $R_{s}$ can result in the development of numerical instabilities.)

A steady state large-signal solution for an rf source current magnitude of $1 \mathrm{~A}$ is illustrated in Figs. 4 and 5. In this particular case, the chamber pressure is $300 \mathrm{mT}$, the electrode radius is $10 \mathrm{~cm}$ and the electrode separation is $2 \mathrm{~cm}$. The discharge is 

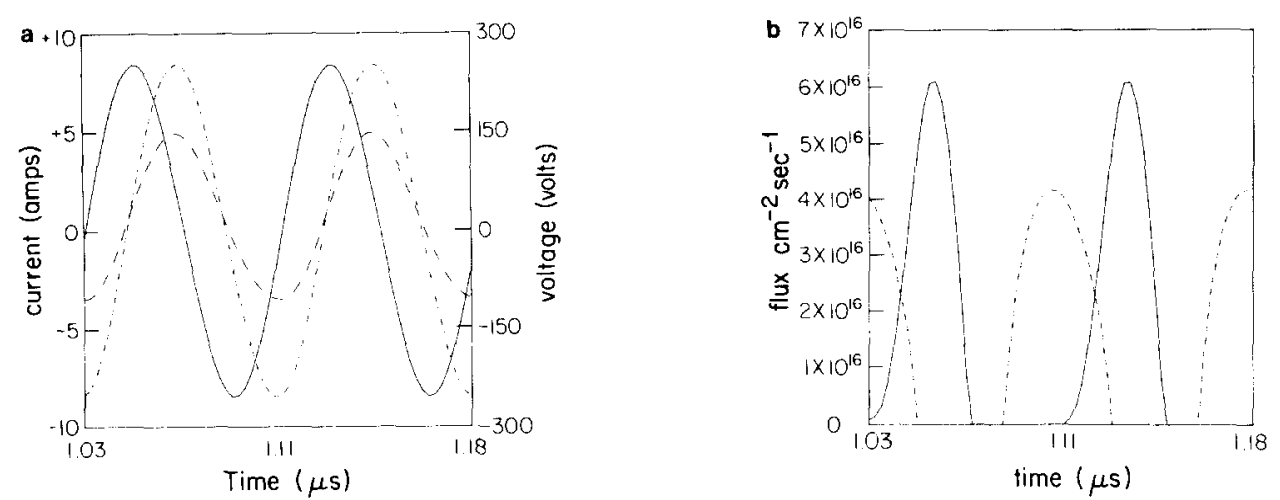

FIG. 4. Results from the large signal if glow discharge simulation for argon at $13.56 \mathrm{MHz}$ and $300 \mathrm{mT}$ : (a) terminal current (solid line), terminal voltage (uneven dashed line) and plasma region voltage (large dashed line); (b) cathode particle fluxes (solid line for electrons and uneven dashed line for ions).

divided into 25 "cells" (i.e., this is equivalent to 25 points with index $j$ and 26 points with index $i$ ). Fig. 4a illustrates the terminal current and voltage waveforms as a function of time. These are Fourier analyzed to obtain the steady state rf power dissipation and the impedance. In this particular case, the rf power dissipation is $129.0 \mathrm{~W}$ and the impedance at $13.56 \mathrm{Mhz}$ is $7.275-j 29.52 \Omega$. From the cathode particle fluxes depicted in Fig. 4b, the number of particles impinging on the cathode per unit time can be calculated. Moreover, averaging the velocity of all incident ions (to the cathode) during an rf cycle yields an average incident $\mathrm{Ar}^{+}$ion energy of $37.62 \mathrm{eV}$. By time-averaging the spatial voltage distribution, the average potential well which confines the electrons in the discharge is obtained (see Fig. 5). (The reason for the hollow in the center of the potential well will be discussed along with convergence criteria later in this section.) The instantaneous dependent variables

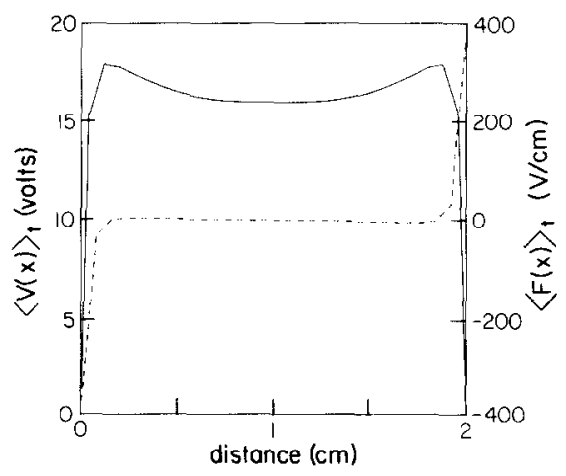

FiG. 5. Time-averaged voltage and field profiles corresponding to simulated results in Fig. 4 (solid line for voltage and uneven dashed line for electric field). 

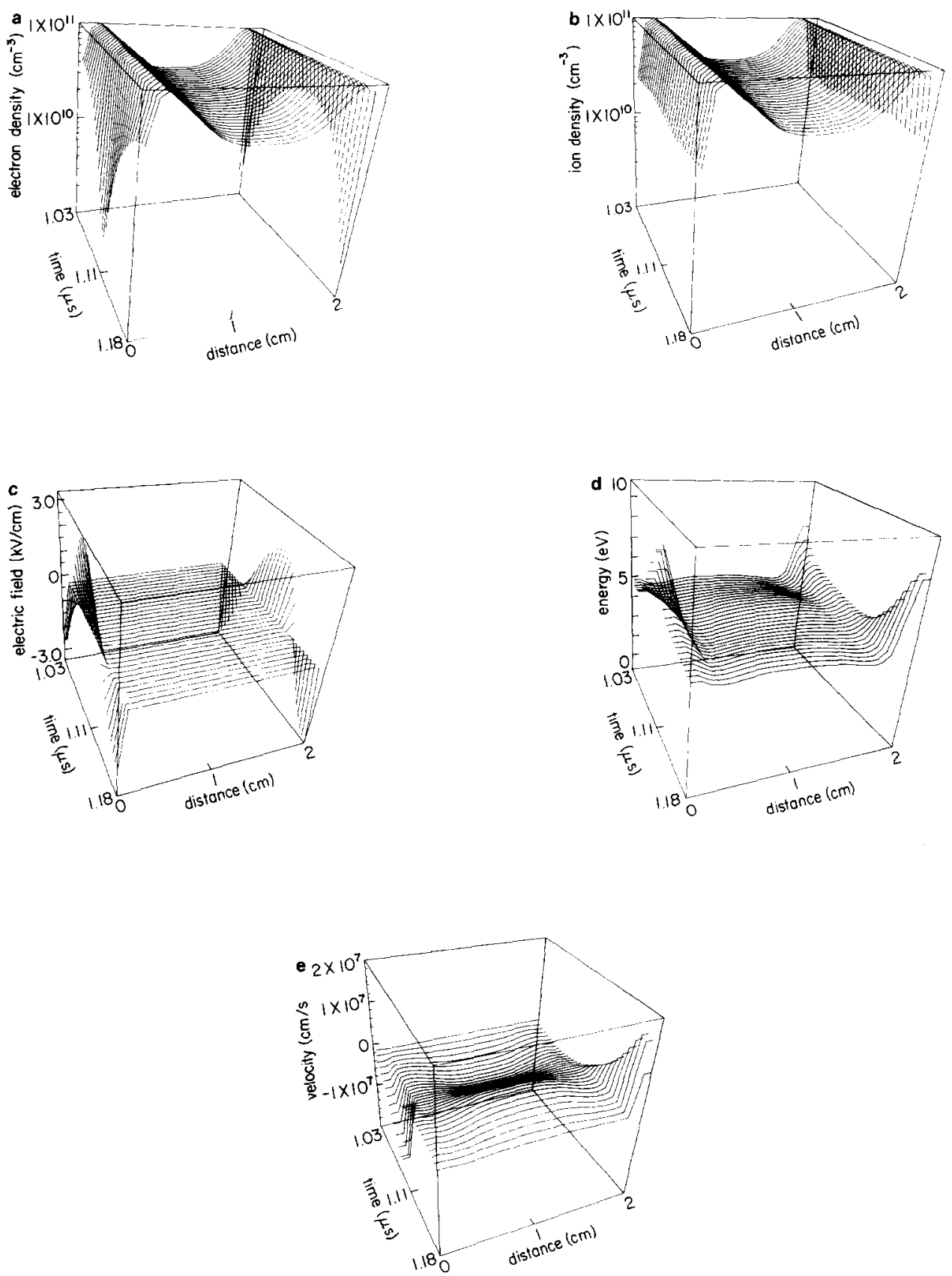

FIG. 6. Dependent variable spatial profiles plotted 32 times during the last simulated $\mathrm{ff}$ cycle: (a) electron density, (b) ion density, (c) electric field, (d) average electron energy, ind (e) average electron velocity. 

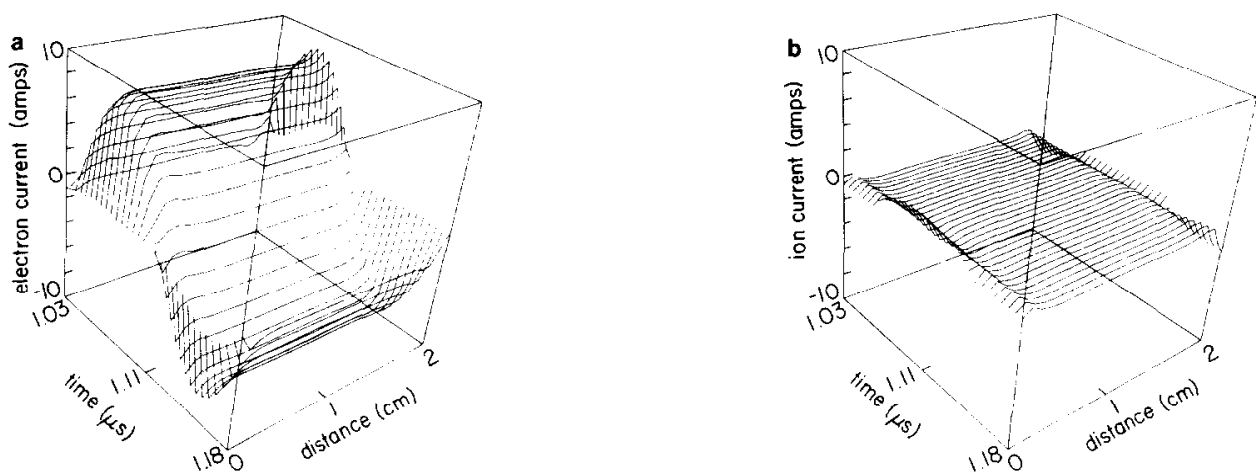

FIG. 7. Particle current spatial profiles plotted 32 times during the last simulated of cycle: (a) electron current and (b) ion current.

can be examined throughout the rf cycle as shown in Figs. 6a-e. The results in these figures corresponds to the second rf cycle in Fig. 4, i.e., $t=1.03$ to $t=1.18 \mu$ s. The electron and ion particle currents are illustrated for the same time period in Figs. 7a, b. As expected, in the center of the discharge or "plasma" region the current is primarily carried by electrons; in the electrode sheath regions there is a significant ion current but most of the current is due to the displacement of the time-varying electric field.

Using the data plotted in Fig. 1a, a metastable excitation rate (see Fig. 8a) is calculated for the same rf period as the ionization rate, depicted in Fig. 8b. (The excitation rate $r_{1}$ is the product of the excitation rate coefficient and the electron density.) Using this rate data, a steady state calculation of the discharge radiation output is performed in an approximate manner by using a quasi-two-dimensional
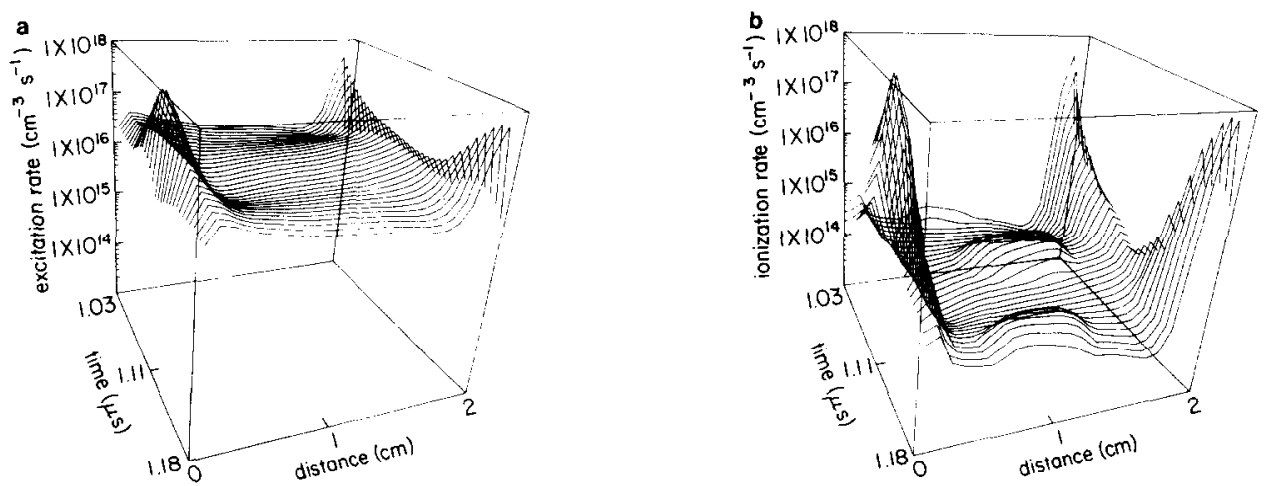

FIG. 8. Electron impact particle generation spatial profiles plotted 32 times during the last simulated rf cycle: (a) excitation rate and (b) ionization rate. 
metastable balance equation which solves the metastable continuity equation along the axis including an approximate loss term due to radial diffusion. This is given by

$$
\frac{\partial M}{\partial t}=-D_{m} \frac{\partial^{2} M}{\partial x^{2}}+r_{1}-D_{m} \frac{M}{A^{2}}-\frac{M}{\tau_{\mathrm{ef}}},
$$

where $M$ is the metastable density, $D_{m}$ the metastable diffusion coefficient, $A$ the radial diffusion length, and $\tau_{\text {eff }}$ the effective radiative lifetime. The diffusion coefficient is estimated to about $100 \mathrm{~cm}^{2} / \mathrm{s}$ by extrapolating the argon ion diffusion coefficient [5] to zero electric field and the diffusion length can be calculated using the expression $A=R / 2.405$, where $R$ is the reactor cylinder radius [35]. Finally, the effective radiative lifetime is calculated with the expression used in Ref. [21] to be $3.24 \times 10^{-5} \mathrm{~s}$. Using a tridiagonal solver to implicitly solve Eq. (28) for $10^{4} \mathrm{rf}$ cycles, the halftone plot of the radiation output (i.e., $P_{\text {rad }}=M \varepsilon_{1} / \tau_{\text {eff }}$ ) in Fig. 9 is generated. The radiation output is constant in time as expected since the radiative lifetime used is several orders of magnitude larger than the rf period. Furthermore, the regions of brightness correspond to those observed in experimental rf glow discharges [36].

The numerical model has been developed in order to achieve a large-signal steady state solution. The criteria for a steady state are twofold:

1. The voltage and current waveforms are Fourier analyzed every two rf cycles. The rf power and impedance are then calculated at the fundamental (i.e., $13.56 \mathrm{MHz}$ ) and checked against the values from the previous two rf cycles. When the percent difference is less than $5 \%$, this criterion is satisfied.

2. The net charge impinging on the cathode is checked during each if cycle. For a steady state, the total number of electrons and ions striking the cathode during one complete of cycle must differ by less than $1 \%$.

In the actual computer model, the impedance/power criterion is checked first; the net charge is examined once this condition has been satisfied.

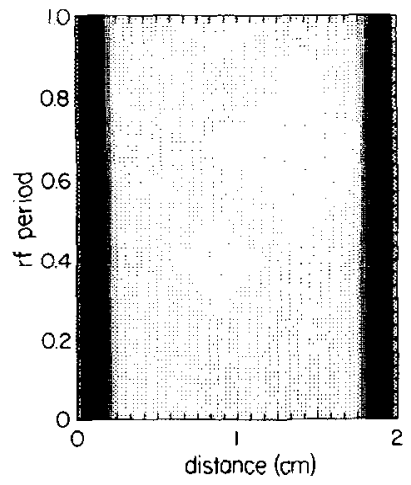

Fig. 9. Haiftone plot of the radiation output for an if cycle. A sixteen level scale is used where the darkest regions correspond to $1.83 \times 10^{18}$ and the lightest regions to $3.64 \times 10^{17} \mathrm{ev} \mathrm{cm}^{-3} \mathrm{~s}^{-1}$. One if period equals $73.7 \mathrm{~ns}$. 
Other more stringent convergence measures could be utilized but the criteria outlined in the previous paragraph are adopted since they are relatively straightforward to evaluate numerically and have been found to represent a point at which the dependent variables no longer vary significantly from one rf cycle to the next. One of the problems with these criteria is evident in Fig. 5 whereby the hollow in the middle of the time-averaged voltage profile indicates that ions are being directed into the center of the discharge and particle number is not being conserved. A more thorough examination reveals that there is a net (equal) flow of both electrons and ions as shown in the plot in Fig. 10 of the time-averaged particle currents. These currents have little effect on the particle density and other dependent quantities. If, on the contrary, there was a net space charge developing in some region as a result of these currents then more rigorous conditions for convergence would be necessary. These currents could be attributed to particle loss due to radial ambipolar diffusion or to volume recombination loss but these are not included in this study since it is felt they are second-order effects. For capacitively coupled $\mathrm{rf}$ glow discharges where the electrode separation is less than approximately half the electrode radius, it has been shown in previous work that radial ambipolar diffusion loss has no significant effect on particle densities [5].

The timestep advancement of this simulation is performed in a multistep manner to facilitate computational efficiency as well as satisfy the stability conditions (26) and (27). At the start of the simulation, a "global" timestep is set to the rf period divided by 64. Before updating any of the dependent variables, the CFL condition (26) is computed. The "global" timestep is then subdivided, if necessary, into "local" timesteps which satisfy the CFL condition. This process is then repeated every "local" timestep for the dielectric relaxation timestep constraint. As a result, the actual timestep used in the finite difference equations can be several orders of magnitude less than the original "global" timestep. The execution time for the results presented in Figs. 4-10 was approximately $30 \mathrm{~h}$ using FORTRAN 77 on an Apollo Domain Series 3000 workstation.

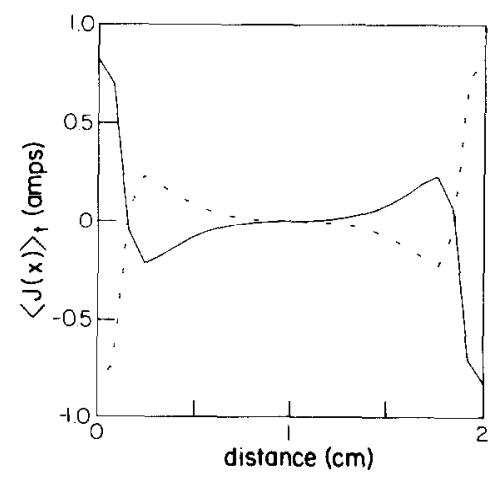

Fig. 10. Time-averaged particle currents corresponding to simulated results in Fig. 4 (solid line for electron current and uneven dashed line for ion current). 


\section{Concluding Remarks}

The motivation for the development of the numerical model described in this paper arises from the need to quantitatively understand the physical/chemical mechanisms in microelectronic plasma chemical processing. In particular, the energy-momentum electron transport model is used to facilitate the understanding of the nonequilibrium electron effects which other models $[5,6]$ are unable to predict due to their governing assumptions. The finite-difference formulation of the self-consistent rf glow discharge model (consisting of the continuity equations for clectrons and ions, the energy and momentum conservation equations for electrons, and Poisson's equation) poses a serious numerical stability problem. Its numerical complexity is further augmented by the need to preserve the transportive property for all advected quantities as well as maintain strict charge conservation to implicitly satisfy Gauss's law. The resultant numerical scheme utilizes several finitedifference operators, a staggered spatial grid, and a method of subdividing the timestep to alleviate these problems. Future work by the authors involving if glow discharge modeling will include a thorough comparison of this model with a quasistatic "drift-diffusion" model [5] to investigate nonequilibrium electron effects and the development of experiments to further examine the model's validity. Moreover, the potential for utilizing a multi-group electron model [21] to include electronelectron and superelastic collisions will be investigated.

\section{APPENDIX: LIST OF SyMBOLS}

$\partial, \quad$ the single variable differential operator

$\nabla, \quad$ the gradient operator

$f, \quad$ the particle distribution function

$v, \quad$ the general particle vector velocity; later redefined as the ion drift velocity

$\bar{F}, \quad$ the external force exerted on a particle

$m, \quad$ the electron rest mass

$\bar{r}, \quad$ the particle position

$t$, the time

$n$, the electron density

$u$, the electron average velocity

$w, \quad$ the electron average energy

$P, \quad$ the electron pressure

$k_{b}, \quad$ Boltzmann's constant

$T, \quad$ the electron temperature

$\alpha, \quad$ the electron impact ionization rate

$\tau_{v}, \quad$ the electron effective momentum relaxation time

$\tau_{w}, \quad$ the electron effective energy relaxation time

$w_{0}, \quad$ the thermal energy associated with the neutral gas reservoir 


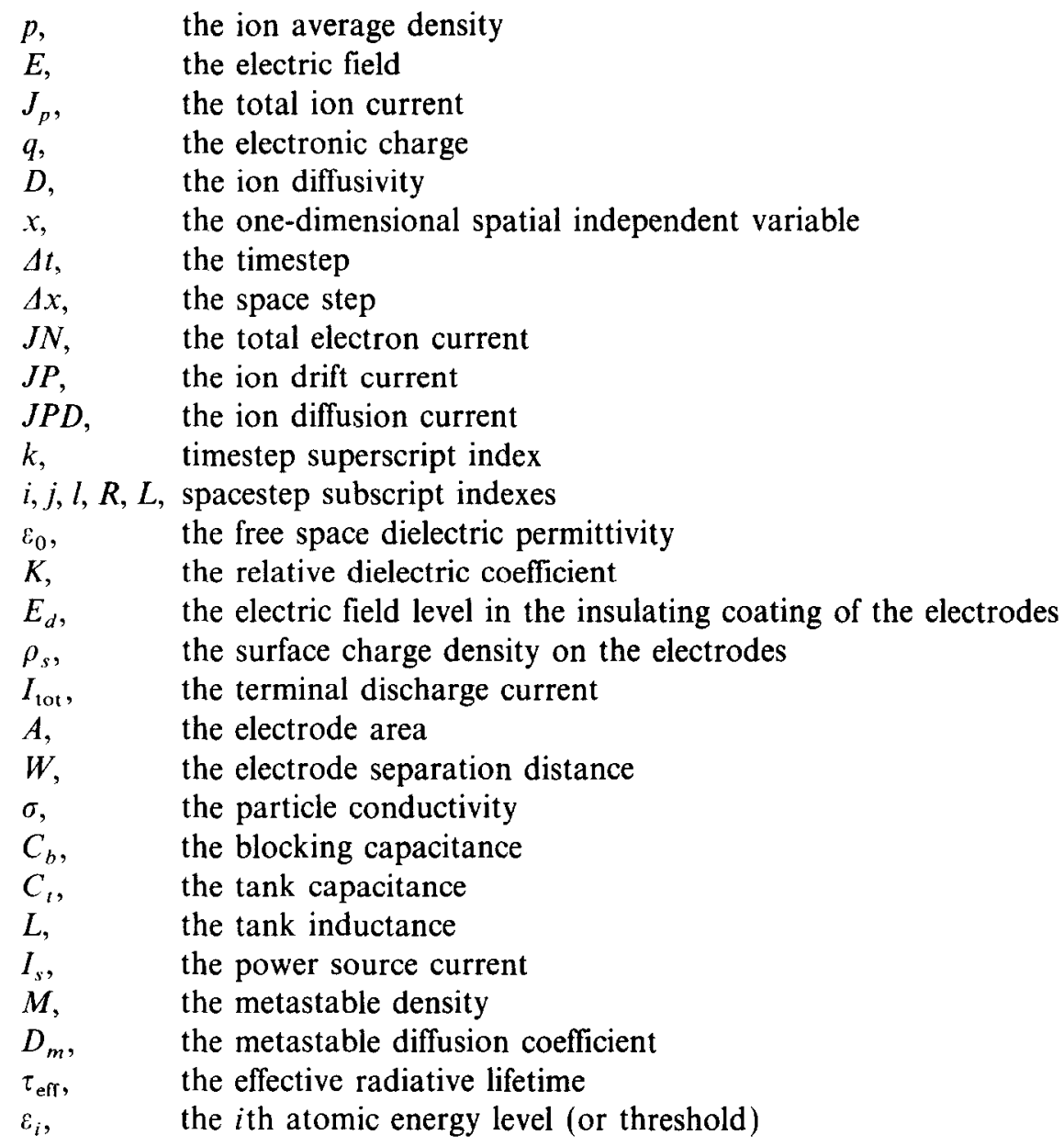

\section{ACKNOWLEDGMENT}

This work is being supported by the Semiconductor Research Corporation (SRC) under Contract 86-07-085.

\section{REFERENCES}

1. J. W. CoBurn, Plasma Etching and Reactive Ion Etching (American Vacuum Society Monograph Series, American Institute of Physics, New York, 1982).

2. F. J. Kampas, Semiconductors and Semimetals, Vol. 21A (Academic Press, New York, 1984), pp. 153.

3. B. N. Chapman, Glow Discharge Processes (Wiley, New York, 1980).

4. A. L. Ward, J. Appl. Phys. 33, 2789 (1962).

5. M. S. Barnes, T. J. Cotler, ANd M. E. Elta, J. Appl. Phys. 61, 81 (1987). 
6. D. B. Graves and K. F. Jensen, IEEE Trans. Plasma Sci. PS-14, 78 (1986).

7. T. ITOH AND T. Musha, J. Phys. Soc. Japan 15, 1675 (1960).

8. R. W. L. Thomas and W. R. L. Thomas, J. Phys. B 2, 562 (1969).

9. N. A. Tran, E. Marode, and P. C. Johnson, J. Phys. D 10, 2317 (1977).

10. M. J. Kushner, J. Appl. Phys. 54, 4958 (1983).

11. W. L. MORGAN, JILA Information Center Report No. 19, JILA, University of Colorado, Boulder, 1979 (unpublished).

12. R. M. Thomson and A. R. Davies, Comput. Phys. Commun. 11, 369 (1976).

13. K. Huang, Statistical Mechanics (Wiley, New York, 1963).

14. R. K. Froenulch, Ph.D. thesis, University of Michigan, Ann Arbor, 1982 (unpublished).

15. M. Mitchner And C. H. Kruger, JR., Partially loized Gases (Wiley-Interscience, New York, 1973).

16. W. P. Allis, Encycopedia of Hhysics, Vol. 21 (Springer-Verlag, Berlin, 1956), p. 383.

17. I. P. Sharofsky, T. W. Johnston, and M. P. Bachynski, The Particle Kinetics of Plasmas (Addison-Wesley, Reading, MA, 1966).

18. E. NASSER, Fundamentals of Gaseous Ionization and Plasma Electronics (Wiley-Interscience, New York, 1971).

19. G. Francis, Encyclopedia of Physics, Vol. 22 (Springer-Verlag, Berlin, 1956), p. 53.

20. M. J. Druyvesteyn and F. M. Penning, Rev. Mod. Phys. 12, 87 (1940).

21. L. VRIENS, J. Appl. Phys. 44, 3980 (1973).

22. L. VRiens and A. H. M. Smeets, Phys. Rev. A 22, 940 (1980).

23. L. J. KIEFFER, JILA COM-74-11661, JILA, University of Colorado, Boulder, 1973 (unpublished).

24. K. BLOTEKJAER, IEEE Trans. Electron Devices ED-17, 38 (1970).

25. P. J. ROACHE, Computational Fluid Dynamics (Hermosa, Albequeque, NM 1982).

26. P. D. LaX, Commun. Pure Appl. Math. 7, 159 (1954).

27. R. D. RichtmeYER, NCAR Technical Note 63-2, Boulder, CO, 1963 ,

28. R. W. MaCCormack, in Proceedings, 2nd Intl. Conf. on Numerical Methods in Fluid Dynamics, Lecture Notes in Physics (Springer-Verlag, New York, 1971).

29. H. J. Longley, LASL Report LAMS-2379, Los Alamos Scientific Lab., Los Alamos, NM, 1960.(unpublished).

30. P. E. Bauhahn, Ph.D. thesis, University of Michigan, Ann Arbor, 1977 (unpublished).

31. R. A. Gentry, R. E. Martin, and B. J. Daly, J. Comput. Phys. 1, 87 (1966).

32. R. Courant, E. Isaacson, And M. Rees, Commun. Pure Appl. Math. 5, 243 (1952).

33. W. H. HAYT, JR., Engeneering Electromagnetics (McGraw-Hill, New York, 1967).

34. C. M. LEE, Ph.D. thesis, University of Michigan, Ann Arbor, 1974 (unpublished).

35. B. E. Cherrington, IEEE Trans. Electron Devices ED-26, 148 (1979).

36. P. Bletzinger and C. A. DeJosePh, JR., IEEE Trans. Plasma Sci. PS-14, 124 (1986). 